fique international

Université fédérale

Gianpaolo Baiocchi

vnl : Luiggi Bobbio IUni.uc Boltanski (EHESS); le des Mines):

Université de Leeds] : arta (Institut Universiohn Dryzek Université э Eliasoph [Université id] ; Joan Font (IESA/ raser lthe New School ] ; John Gastil [Univer;Brigitte Geissel de Francfort] ; Pierre de Montréal] ; William ¿Wisconsinl ; Les versityl: Dominique Ju Québecà Montréal] ; Université Catholique ard Manin (EHESS) ;

Iniversité d'Harvard! ; versité d'Ohiol ; Chanité de Westminsterl: sité de Pennsylvaniel: os lUniversité de ique Pestre [EHESS] ; (Collège de France) ; iversité de Poitiers) ; Université Libre de :einer IUniversité de Michael Schudson mbial ; Gerry Stoker hamptonl; Lawrence Mark E. Warren [Uni? Britanniquel; Brian Je Lancasterl.

\title{
a revue
}

cipations.fr

Groupement d'intérêt :ipation du public, atie participative."

\section{mocratie jarticipation}

at-democratie.fr

\section{Dynamiques de la participation en Amérique latine}

revue de sciences sociales sum de democratte et la citoyenneté 


\section{Dossier : Dynamiques de la participation en Amérique latine}

\section{Coordonné par David Garibay, David Recondo, Héloïse Nez}

Introduction du dossier. Vingt-cinq ans après Porto Alegre, où en est [l'étude de] la démocratie participative en Amérique latine?

David Garibay.

Rituels démocratiques et mise en scène de la participation populaire dans les assemblées du budget participatif à Recife (Brésil)

Camille Goirand

Décloisonner la comparaison : dispositifs participatifs et démocratisation au Brésil et au Mexique

Françoise Montambeault

La diffusion des modèles de démocratie participative au niveau local au Salvador : les maires au carrefour de conceptions concurrentes David Garibay. .......................................... 115

Participer dans les scènes ritualisées du pouvoir.

La Banca Abierta et le Concejo en los Barrios en Argentine Rocio Annunziata

Le défi participatif : délibération et inclusion démocratique dans les budgets participatifs

Ernesto Ganuza, Francisco Francés. 


\title{
Le défi participatif : délibération et inclusion démocratique dans les budgets participatifs
}

\author{
> Ernesto Ganuza \\ > Francisco Francés \\ > Traduction de l'espagnol : David Garibay
}

n en France qu'à tous les domaines zritiques, comptes es grandes revues et étudiants, mais de la vie politique,

ions)

liers)

laction

@sciencespo.fr

495186

\section{> Résumé}

La théorie démocratique a connu ces dernières années un tournant communicationnel, qui a favorisé l'organisation d'expériences participatives reposant sur certains principes de la théorie délibérative, et notamment ceux d'inclusion et d'égalité des participants à la délibération. Le développement des budgets participatifs au cours des dix dernières années offre un excellent cadre analytique pour tester dans quelle mesure ces expériences mettent en œuvre les principes d'inclusion et de participation égalitaire à la délibération. Cet article cherche à contextualiser l'analyse des budgets participatifs, en prenant en compte leur signification et la façon dont ils se déroulent, à partir d'une recherche comparée au Brésil, en Espagne, en France et en Allemagne. Il s'agit en particulier d'interroger l'opposition ou la tension faite dans la théorie politique entre participation et délibération. Il est évident que la délibération demande des espaces de réflexion que les dispositifs participatifs ne peuvent pas offrir. À ce titre, les budgets participatifs visent à concilier ces deux dimensions. Lobjectif de ce papier est ainsi de comprendre les différences entre ces dispositifs selon le contexte politique dans lequel ils se déploient. Les résultats de cette recherche permettent de mieux comprendre comment articuler les différentes procédures mises en œuvre et quelle est la relation entre participation et délibération. 


\section{Le défi brésilien}

En 1989, le gouvernement de la ville de Porto Alegre au Brésil a lancé une expérience politique, le budget participatif, dont le modèle s'est diffusé en moins de 25 ans sur les cinq continents [Sintomer et al., 2010]. L'exemple brésilien a constitué un défi aux formes contemporaines de gouvernement, en faisant converger la tradition politique de la gauche latino-américaine avec de nouvelles formes daction politique qui proviennent des nouveaux mouvements sociaux et du tournant délibératif de la théorie politique [Baiocchi, Ganuza, 2014l. Le budget participatif intègre l'importance croissante de la communication politique. marque une distance avec les pratiques autoritaires et ouvre les processus de légitimation politique à des procédures d'influences réciproques. Mais surtout, le budget participatif a permis l'inclusion dans le jeu politique d'individus perçus comme des agents rationnels qui réfléchissent à partir de leurs préférences dans le cadre d'un débat ouvert, où chacun d'entre eux a le droit d'être informé, de prendre la parole et d'être écouté au même titre que les autres. A contre-courant de la gauche latino-américaine, le gouvernement du Parti des travailleurs à Porto Alegre a rompu avec l'idée d'un gouvernement reflet de la société, en assumant la pluralité des préférences qui structurent la société. l'existence de conflits entre elles et en les intégrant dans un cadre politique fondé sur des idées universelles comme celle de délibération (Baiocchi, Ganuza, 2014l.

Le budget participatif de Porto Alegre a été qualifié de «best practice » par le PNUD en 1996. Jusqu'en 2004, il était caractérisé par un cadre institutionnel marqué par la communication et par la mise en œuvre de politiques publiques fondées sur un critère de justice sociale (Baiocchi, 2005). Tout cela a permis d'inscrire dans la durée une nouvelle manière de gouverner qui a traversé de nombreuses frontières et a été appliquée dans des contextes très différents en termes de développement, de culture ou de tradition politique (Sintomer et al., 2010l. La Banque mondiale a qualifié le budget participatif de stratégie efficace dans la lutte contre la pauvreté (Goldfrank, 2012), ce qui a contribué à sa diffusion en Afrique et en Asie. En Europe, la diffusion de ce dispositif a été davantage liée à une rhétorique de modernisation administrative [Sintomer et al., 2008), mais en reprenant les mêmes arguments : la recherche d'une nouvelle manière de gouverner inspirée de principes de communication et fondée sur des procédures qui permettent à tous les citoyens de participer.

Ce nouveau dispositif institutionnel a permis un dialogue entre les élites politiques et économiques et le public hors des périodes électorales. Il met en avant la fonction délibérative au sein des administrations, en ayant comme objectif la définition collective des politiques publiques. Mais le budget participatif fait face également à des défis importants. Il incarne une démarche constructive, dans lequel les citoyens présentent leurs projets et les priorisent au sein d'espaces participatifs établis pour cela, démarche opposée à la tradition de contestation qui a davantage caractérisé les formes de participation de la gauche. La 
ncé une expéusé en moins aple brésilien nt, en faisant avec de noumouvements cchi, Ganuza, I communica$s$ et ouvre les - réciproques. jeu politique nt à partir de entre eux a le e titre que les rernement du ouvernement structurent la in cadre poliion (Baiocchi,

ctice » par le institutionnel ies publiques ela a permis a traversé de différents en ntomer et al. tégie efficace ué à sa diffuité davantage et al. 2008\}, relle manière Ir des procé-

$s$ élites polimet en avant ne objectif la patif fait face ructive, dans in d'espaces de contestaa gauche. La participation suppose donc de rompre avec des cadres classiques et d'inclure des éléments de communication. Au lieu de donner place aux formes traditionnelles de lobbying pour la défense d'intérêts particuliers, les dispositifs participatifs établissent des modes de fonctionnement qui permettent un dialogue ouvert entre les intérêts en conflit. La relation entre gouvernants et gouvernés devient plus horizontale [Sintomer et al., 2008). Ces caractéristiques conduisent la participation vers un nouveau cadre dans lequel la reconnaissance des différences dans les débats joue un rôle essentiel, ce qui renforce l'égalité des citoyens au moment de prendre la parole. Les travaux développés au Brésil sur le budget participatif ont montré que son succès ne relève pas seulement des facteurs avancés par les théories néo-tocquevilliennes, pour lesquelles l'élément déterminant serait le degré de participation antérieure des citoyens, mais qu'il est dû aussi à une dimension institutionnelle que Leonardo Avritzer (2006) appelle l'efficience du processus délibératif, c'est-à-dire au fait que la participation perdure et a du succès si les citoyens peuvent effectivement influencer les politiques publiques. Gianpaolo Baiocchi [2005] et Brian Wampler [2007] vont dans le même sens lorsqu'ils soulignent l'importance des conditions et qualités des institutions locales dans l'analyse de ces expériences, qui peuvent être évaluées à l'aune de leur efficacité administrative lexécution effective des propositions débattues par les citoyensl et de l'inclusion large de l'ensemble des citoyens Idistribution égalitaire des opportunités pour chaque citoyen de délibérer et influencer).

Cet article cherche à analyser ces nouveaux dispositifs dans l'objectif d'évaluer si l'on peut effectivement observer, d'une part, l'inclusion d'opinions diverses et, d'autre part, la distribution égalitaire des opportunités de délibération entre les participants. Nous aborderons les cas brésiliens en interrogeant le profil des citoyens qui se mobilisent dans les assemblées participatives et leur degré d'implication dans les dynamiques de délibération. Les résultats permettront de discuter la pertinence de la délibération comme variable politique à analyser, un point qui fait l'objet d'importants débats (Mutz, 2006 ; Hibbing. Theiss-Morse, 2002l. Pour cela, nous nous appuierons sur une étude quantitative réalisée dans huit villes situées dans quatre pays (Brésil, Espagne. France et Allemagnel. Cela nous permettra de considérer l'importance des variables contextuelles dans chaque cas, et l'influence des types de dispositifs mis en œuvre dans l'un ou l'autre des pays. Dans un premier temps, nous reviendrons sur le défi politique que représente le budget participatif. Nous présenterons ensuite les dispositifs délibératifs mis en place dans les expériences que nous allons comparer. Puis, à partir du profil des participants, nous évaluerons le degré d'inclusion de chaque expérience. Enfin, nous analyserons les dispositifs délibératifs et les résultats auxquels a conduit chaque type de configuration institutionnelle. 


\section{Le problème de l'inclusion et de la délibération dans un gouvernement participatif}

L'idée de donner davantage de pouvoir aux citoyens suppose généralement un accroissement de leurs capacités, afin qu'ils puissent réellement participer aux tâches de gouvernement: Un des grands défis à cet égard est le problème de l'inclusion, c'est-à-dire dans quelle mesure les participants peuvent effectivement développer des capacités communicationnelles leur permettant de s'intégrer à un débat ouvert sur les activités du gouvernement. Or l'enjeu ne réside pas seulement dans la mise en place d'un dispositif qui aurait une logique politique distincte [de nature «propositive »] : en effet, dans ces expériences le gouvernement conserve une place prépondérante dans l'organisation même des processus participatifs - aspect politiquement très sensible, car ces dispositifs reposent sur l'idée centrale d'une inclusion large et diversifiée des citoyens dans ces expériences, et de leur capacité à intervenir dans les affaires publiques.

Les travaux académiques montrent combien les dispositifs participatifs présentent de nombreuses limites quant à l'inclusion large et diversifiée des citoyens. Ceux qui participent ont habituellement à la fois un niveau de formation et un intérêt pour la politique plus élevés que la moyenne (Verba et al., 1995 ; Bryan, 2004]. Or, dans un cadre délibératif, de nouveaux problèmes surgissent : aux limites habituelles de la participation, s'ajoute la question des compétences communicationnelles. Pour beaucoup d'auteurs, l'inclusion large et diversifiée des citoyens est fortement limitée par les conditions d'une influence active au cours d'une expérience publique [Fiorina, 1999 ; Sanders, 1997]. Diana Mutz (2006) va plus loin en suggérant que l'existence de dispositifs participatifs suppose l'acceptation par chacun du point de vue de l'autre, un élément que l'on ne retrouve pas habituellement chez les citoyens qui participent activement en politique, car ces derniers agissent plutôt de telle sorte à obtenir la satisfaction de leurs intérêts propres et font partie de réseaux sociaux homogènes. Nina Eliasoph [1998] a montré combien les individus fortement impliqués dans des activités bénévoles dans le monde associatif aux États-Unis ont tendance à éviter de parler de politique en public, ce qui réduit d'autant leur propre confrontation à d'autres points de vue.

Les problèmes d'inclusion et de délibération sont d'autant plus importants que le dispositif participatif analysé dans cet article, le budget participatif, ne constitue pas un mécanisme institutionnel favorisant un niveau intense de délibération. D'autres dispositifs comme les jurys citoyens ou les sondages délibératifs sont très attentifs à cet aspect : le recrutement se fait par tirage au sort, ce qui garantit l'hétérogénéité du profil des participants, et la délibération est fortement structurée, ce qui maintient une distribution effective des opportunités de délibération parmi les participants [Barabas, 2004]. Or, dans les budgets participatifs, il n'y a ni mécanismes particuliers pour s'assurer de la diversité du profil des participants, ni procédures délibératives intensives. Généralement, l'ensemble de la population est invité à participer à ces assemblées, et ceux qui 


\section{ion dans}

ralement un nt participer le problème suvent effecrmettant de 3r l'enjeu ne $t$ une logique périences le. in même des is dispositifs itoyens dans ibliques.

sipatifs préersifiée des Je formation et al., 1995 ; surgissent : ompétences t diversifiée ce active au Diana Mutz :ipatifs supent que l'on tivement en la satisfacgènes. Nina is dans des nce à éviter infrontation

importants ticipatif, ne intense de dages déliige au sort. ération est opportunies budgets a diversité éralement, et ceux qui sont présents peuvent intervenir pour défendre une position, faire une proposition ou questionner ce qui est fait, mais cela ne donne pas lieu à un processus intensif de délibération.

Ce problème est central pour la théorie politique et pour l'action pratique des gouvernements. Linitiative brésilienne du budget participatif modifie la structuration de la politique dans les sociétés contemporaines, en mettant la participation au cœur des activités de gouvernement, loin des propositions habituelles de la gauche politique et du tournant délibératif (Swyngedouw, 2005). D'après Jürgen Habermas (1996), la politique moderne s'articule autour de deux moments : il y aurait tout d'abord une délibération informelle des citoyens hors des institutions dans l'espace public, puis une formalisation de ce débat au sein des organisations politiques et sociales qui influencent les délibérations dans les institutions. Cette articulation repose sur le rôle des organisations collectives, acteurs responsables de la diffusion des demandes informelles au sein de l'espace public, dans le but d'influencer les représentants du système politique. Or l'expérience du budget participatif qui a commencé en 1989 à Porto Alegre repose sur une autre conception : ce qui, dans la perspective habermassienne, consistait en une délibération informelle se structurant dans des débats au sein d'organisations collectives avant d'être transmis au système politique devient une délibération formelle dans l'espace public là travers les mécanismes du budget participatif] qui se traduit par une influence directe des citoyens sur la gestion publique.

Le défi posé par l'expérience brésilienne a été celui de la radicalisation de la démocratie, au sein même des structures représentatives de la politique moderne. Lexpérience a connu un succès considérable, puisqu'en moins de 25 ans des budgets participatifs ont été mis en œuvre à travers la planète et dans des conditions économiques et sociales très diverses. Cette diffusion renforce ainsi l'acuité de la question de la nature de ces budgets participatifs au regard des problèmes d'inclusion et de délibération mentionnés plus haut. Comme le gouvernement local conserve la maîtrise de la mise en place de ces dispositifs. de la promotion du débat et des formes de recrutement des participants, il peut exister des biais importants concernant les caractéristiques de la participation et les modalités de délibération, conduisant potentiellement à l'émergence de formes de domination politique. Nous pouvons par conséquent interroger les limites de ces expériences, en analysant les degrés d'inclusion et les activités délibératives des participants.

Pour cela, nous allons présenter les dispositifs délibératifs proposés dans chacun des cas étudiés, dans l'objectif d'apprécier leurs ressemblances et leurs différences, éléments qui seront pris en compte pour valoriser à la fois la nature de la participation et les opportunités de délibération entre les participants. 


\section{Présentation des dispositifs participatifs et de l'étude réalisée}

Les réflexions présentées ici sont issues d'une recherche réalisée en 2010 et coordonnée par l'Institut d'études sociales avancées du Conseil supérieur des recherches scientifiques d'Espagne. L'enquête a porté sur 17 villes de six pays différents. Une étude exploratoire préalable s'est cependant avérée nécessaire, au vu de l'inexistence d'une recension exhaustive des expériences locales de budgets participatifs et de l'absence d'informations concernant la structure sociodémographique des participants aux budgets participatifs des différentes villes étudiẻes?.

Le choix des villes qui composent l'étude est à la fois stratégique et pragmatique. D'une part, ces huit villes ont été choisies pour la représentativité de leurs expériences dans leurs pays respectifs du point de vue de leur organisation interne, tant en termes de méthode que de structure de la population, ainsi que de leur prolongement dans le temps, ce qui permet de les considérer comme des cas paradigmatiques et intéressants pour l'analyse. D'autre part, le choix de ces cas tient également au nombre plus important de questionnaires recueillis sur ces expériences, ce qui leur confère un niveau de fiabilité plus élevé dans les estimations réalisées à partir de l'exploitation et de l'analyse des données².

Le questionnaire est structuré autour de deux grandes parties. La première porte sur la perception et les opinions des enquêtés concernant les formes conventionnelles de participation lintérêt et suivi de la politique, confiance dans les institutions, connaissance de l'action publique municipale, appartenance à des groupes collectifs organisés, idéologiel. La seconde partie s'intéresse à

[1] Le choix de l'échantillon, en lien avec l'étude exploratoire, a pris en comple les variables du sexe et de l’âge des participants afin de parvenir à un echantillonnage non probabiliste, la nature de l'enquête ayant rendu impossible la définition de lacteurs d'échantillonnage stricts ldu fait de l'inexistence de données sur les caractéristiques d'ensemble de la population de référencel. Le nombre total de questionnaires recuellti est de 1881, dont 918 ont été retenus comme représentatifs des huit cas analysés dans cet article. Les cas finalement retenus ne résultent pas d'un choix aléatoire puisquils ont été autosélectionnés. Le nombre de questionnaires retenu permet néanmoins, en termes d'équivalences pour un calcul statistique représentatif, de parler d'un niveau de confiance de $95,5 \%$ et d'une marge derreur inférieure à $\pm 3,5 \%$, au vu d'une variance inconnue de la population de référence. Si ces éléments niempêchent pas de parler d'inférences statistiques, ils permettent dappuyer une analyse solide des cas analysés.

[2] Le questionnaire utilisé est une version améliorée de celle utilisée lors d'une enquête précédente réalisée en 2007 dans une recherche menếe en Espagne par l'Institut d'études sociales avancées, dont les résultats ont permis de valider une grande partie des indicateurs du questionnaire actuel. Le questionnaire précédent a également été enrichi par des suggestions et des réflexions de chercheurs des différents pays intégrés dans le projet, visant à renforcer la capacité réelle des indicateurs choisis à mesurer la complexité des processus participatifs, ce quí a ainsi permis de fournir une plus grande validité au contenu du questionnaire définitif. Le recueil des questionnaires a été réalisé de la même manière dans les différentes villes à partir de questionnaires anonymes, validés préalablement et auto-administrés, avec l'aide technique des personnes en charge des dispositifs.

let 
2010 et eur des leurs modalités d'implication dans le budget participatif fexpérience, actions réalisées dans le processus, perception des résultats obtenus, etc.].

De manière générale, les budgets participatifs fonctionnent sur l'idée que les citoyens sont invités à participer à l'occasion d'assemblées publiques, dans lesquelles, la plupart du temps, les participants peuvent prendre la parole. Les propositions sont établies avant ou pendant les assemblées, au cours desquelles on opère une priorisation entre elles, établie par le vote et/ou selon des critères de justice sociale. Dans certains cas, des discussions ont lieu, mais pas de choix ni de prise de décision. L'ensemble du dispositif peut avoir un cadre réglementaire explicite, établi par les citoyens ou par l'administration. Un des objectifs de l'étude est de montrer dans quelle mesure les procédures spécifiques à chaque cas ont des effets sur la délibération. Nous savons par des travaux antérieurs qu'il peut exister des biais dans la participation liés au profil socioéconomique des participants, et que ces biais sont atténués par les procédures délibératives (Bryan, 2004 ; Ganuza, Francés, 2012). Mais cela est-il le cas dans tous les pays ? Les procédés délibératifs choisis ont-ils une influence à cet égard?

Les huit cas retenus (Porto Alegre et Guarulhos au Brésil, Ferrol et Novelda en Espagne, le $20^{\circ}$ arrondissement de Paris et Grigny en France, larrondissement de Kreuzberg à Berlin et Postdam en Allemagne] ont chacun leurs particularités, mais peuvent être regroupés suivant la typologie des budgets participatifs proposée par Yves Sintomer et al. (2008). À partir de cas européens, la classification retenue a établi six modèles différents de participation : 1] démocratie participative, 2l modernisation participative, 3) démocratie de proximité, 4 l partenariats public/privé, 5] développement communautaire, 6] néocorporatisme. Les expériences retenues ici correspondent aux trois premiers cas.

Le modèle de la démocratie participative correspond à des contextes habituellement progressistes, où la modernisation administrative n'est pas lobjectif premier. Les dispositifs visent à démocratiser la démocratie, en intégrant les savoirs d'usage des citoyens dans les processus de prise de décision. Il sagit d'expériences avec des procédures bien établies, qui se caractérisent par une intensité de délibération élevée, et par l'existence de critères de justice sociale pour prioriser les propositions débattues. Les citoyens ont une marge de mancuvre dans l'établissement et l'évolution des règles de l'expérience, ce qui leur donne une place particulière dans le dispositif, où ils ne sont pas de simples invités. Le débat participatif s'oriente sur les investissements publics, même s'il peut y avoir des différences sensibles d'un cas à l'autre. En effet, dans les cas européens, il s'agit plutôt de petits investissements urbains, alors que dans les cas brésiliens, l'intervention est plus ambitieuse. Les décisions prises dans ce cadre ont généralement une force exécutoire. Les participants aux assemblées sont à la fois des citoyens non organisés, qui peuvent avoir déjà des formes dengagement actif au sein de leurs communes, et des associations locales. La délibération est ici intensive, car il s'agit d'expériences dont l'objectif est de résoudre des conflits à partir de l'échange d'arguments. 
Le modèle de la modernisation participative est inspiré par les analyses du New public management dans lesquelles la participation est vue comme un instrument au service de l'efficacité administrative. Lidée générale est que l'incorporation des citoyens à la décision peut améliorer les résultats de l'administration. $\mathrm{Ce}$ modèle se fonde sur la recherche d'une plus grande légitimation de l'action publique. En ce sens, les expériences niont pas pour objectif de parvenir à une meilleure redistribution des biens publics sur des critères de justice sociale, mais plutôt de contribuer à la paix sociale. Les dispositifs qui s'inscrivent dans ce modèle associent la population aux choix budgétaires, mais seulement dans un cadre consultatif. Les décisions finales sont toujours du ressort des représentants élus. Les règles de fonctionnement ne sont pas toujours bien définies et le niveau de délibération est faible. Les participants sont des citoyens, souvent désignés par tirage au sort, et des associations.

Le modèle de la démocratie de proximité a pour objectif de renforcer l'aspect délibératif des instances représentatives, sans pour autant transposer les modes de fonctionnement de la démocratie représentative dans les espaces participatifs. Il s'agit d'incorporer les savoirs d'usage des citoyens pour renforcer la capacité de gestion de représentants élus au niveau des quartiers. Ici, ce n'est pas la justice sociale qui prévaut mais la solidarité, ce qui ne suppose pas forcément une participation active des habitants. Les procédures mises en place sont consultatives et fondées sur une écoute sélective [Sintomer et al., 2008, p. 77] dans laquelle les participants interviennent et les élus écoutent. Les règles de fonctionnement sont généralement informelles et peu claires, ce qui ne donne pas à la délibération une intensité forte. Les participants sont à la fois des citoyens, actifs ou non, et des associations.

Les huit cas abordés ici peuvent être classés à partir des trois modèles précédents. Pour les cas européens, les deux villes espagnoles relèvent du modèle de la démocratie participative. Mais ce sont les villes brésiliennes qui constituent les cas de référence pour ce modèle : elles représentent des cas paradigmatiques, où l'on retrouve tous les éléments signalés, alors que ce n'est pas le cas des villes espagnoles. Si, dans un premier temps, les deux villes allemandes relevaient du modèle de la modernisation participative, en réalité il nous semble plus pertinent de les situer à mi-chemin entre modernisation participative et démocratie de proximité (Herzberg, 2011). Les deux villes françaises présentent des fonctionnements différents : le $20^{\mathrm{e}}$ arrondissement de Paris correspond bien au modèle de la démocratie de proximité $[\mathrm{Nez}, 2010)$, alors que celui de Grigny est à mi-chemin entre ce modèle et celui de la démocratie participative.

La figure 1 situe les huit cas d'étude par rapport aux trois modèles de participation. En prenant en compte les différences en termes de fonctionnement des dispositifs institutionnels des budgets participatifs et leurs dynamiques internes, la réflexion va désormais chercher à comprendre dans chaque cas les formes de participation et leur intensité délibérative. 
jes du New instrument :orporation tration. Ce de laction venir à une ce sociale, -ivent dans ment dans des repréen définies is, souvent

spect déli; modes de rticipatifs. a capacité pas la jusáment une nt consul. 77) dans $s$ de foncdonne pas ; citoyens,

es précénodèle de onstituent Iradigmapas le cas lemandes is semble ipative et résentent pond bien de Grigny 3.

e particiment des internes. ormes de
Figure 1. Modèles de budgets participatifs et cas étudiés

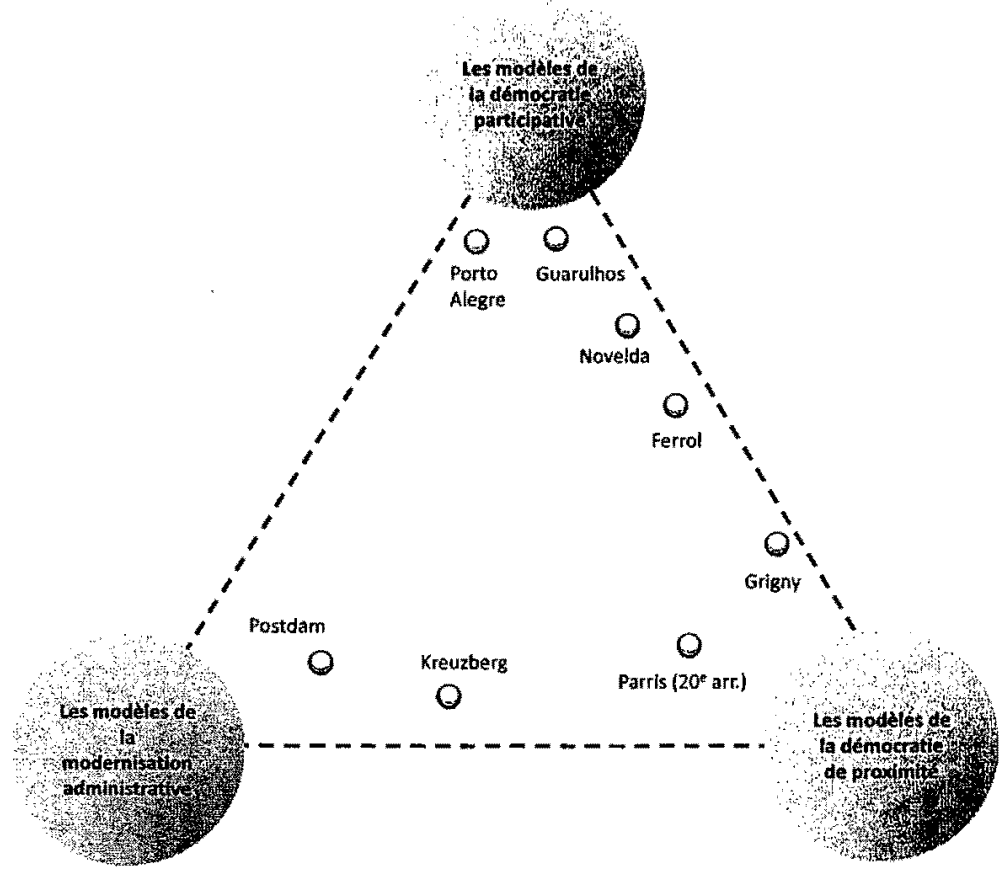

\section{Le degré d'înclusion et la distribution des opportunités délibératives}

Le degré d'inclusion dans un budget participatif permet d'évaluer si les structures sociodémographique et sociopolitique des villes concernées se retrouvent dans les mêmes proportions parmi les participants aux assemblées. Ces écarts peuvent produire des effets incitatifs ou inhibant sur la participation de certains habitants, créant ainsi des biais dans la participation et orientant, le cas échéant, l'espace délibératif créé pour le débat.

Le tableau 1 indique des différences entre les populations des villes étudiées et les participants, en termes sociodémographiques et sociopolitiques. Les différences entre les villes étudiées peuvent être liées à des facteurs externes à ceux évoqués jusqu'à présent, mais permettent tout de même d'ébaucher une comparaison entre elles. Les variations relatives au sexe ne semblent pas significatives. On constate en revanche, dans tous les cas considérés, des écarts importants concernant les variables liées à l'âge et au niveau de formation. Dans tous les cas, les populations de tranches d'âge intermédiaire sont surreprésentées par rapport aux plus jeunes et aux plus âgés. De même, les personnes qui ont un niveau de formation plus élevé que la moyenne sont également surreprésentées, alors que celles de niveau de formation moins élevé sont sous-représentées. 
Tableau 1. Influence des variables sociodémographiques et sociopolitiques dans l'inclusion au sein du budget participatif (présence dans les assemblées citoyennes)

\begin{tabular}{|l|c|c|c|c|c|c|c|c|}
\hline & \multicolumn{2}{|c|}{ ESPAGNE } & \multicolumn{2}{c|}{ BRÉSIL } & \multicolumn{2}{c|}{ FRANCE } & \multicolumn{2}{c|}{ ALLEMAGNE } \\
\hline & Ferrol & Novelda & P. Alegre & Guarulhos & $\begin{array}{c}\text { Paris } \\
200^{\mathrm{E}} \\
\text { arr. }\end{array}$ & Grigny & Kreuzberg & Postdam \\
\hline Sexe & Faible & Forte & Forte & Faible & Forte & Forte & Forte & Forte \\
\hline $\begin{array}{l}\text { Âge } \\
\text { de formation }\end{array}$ & Moyenne & Forte & Moyenne & Forte & Forte & Forte & Moyenne & Forte \\
\hline $\begin{array}{l}\text { Autopositionne- } \\
\text { ment idéologique }\end{array}$ & Moyenne & Faible & Moyenne & Moyenne & Forte & Forte & Forte & Forte \\
\hline $\begin{array}{l}\text { la politique pour } \\
\text { Expérience dans } \\
\text { le processus* }\end{array}$ & Forte & Forte & Forte & Forte & Forte & Forte & Forte & Forte \\
\hline $\begin{array}{l}\text { Participation à } \\
\text { des associations }\end{array}$ & Forte & Forte & Forte & Forte & Forte & Forte & Forte & Forte \\
\hline
\end{tabular}

rhétoriqu élargir di

Au-delà gories at montrenl en compt Au contri écarts, lt avec des qui indiq! médiaire intérêt $m$

Après le délibérat La délibé autorités dures $\mathrm{pr}$ assemble selon diff

J'ai compr nement de J'ai particí en demand et en intert J'ai fait des $J$ 'ai contrib détailler ot une propos par quelqu $J$ 'ai encour participant proposition

$\mathrm{Ne}=918$

Source: IES confirmé plus récemment dans les études sur les Town Meetings aux États-Unis [Bryan, 2004]. Alors que les expériences de budget participatif reposent sur une 


\begin{tabular}{|l|l|}
\hline uzberg & Postdam \\
\hline orte & Forte \\
\hline renne & Forte \\
\hline irte & Forte \\
\hline irte & Forte \\
\hline irte & Forte \\
\hline ille & Forte \\
\hline rte & Forte \\
\hline
\end{tabular}

5 des particitional pour la de 10 points, t5.

articipants et lation la plus

Iur le Brésil :

oyens qui issus de icé. En ce is et ceux parmi les semblées elui de la it déjà été IVerba et litique, et tats-Unis it sur une rhétorique égalitaire, les cas étudiés ici montrent quills ne sont pas parvenus à élargir de manière conséquente le profil des participants.

Au-delà de ces points communs, il est possible d'établir deux grandes catégories au sein des cas étudiés. Les budgets participatifs allemands et français montrent des écarts très significatifs pour quasiment toutes les variables prises en compte entre le public présent et le profil de la population dans son ensemble. Au contraire, dans les cas brésiliens et espagnols, même si on retrouve certains écarts, le profil des participants semble plus diversifié. Il y a toutefois partout. avec des degrés plus ou moins marqués, un écart de participation significatif qui indique que le profil type du participant est celui d'une personne d'âge intermédiaire, avec un niveau de formation avancé (baccalauréat ou plus) et avec un intérêt marqué pour la politique, ce qui introduit des biais considérables.

Après le degré d'inclusion, le deuxième facteur analysé est celui du niveau de la délibération des participants aux assemblées citoyennes du budget participatif. La délibération se déroule à l'occasion des assemblées, qui sont voulues par les autorités comme un lieu formel de structuration des débats. Même si les procédures précises de fonctionnement peuvent varier d'un cas à l'autre, toutes ces assemblées se caractérisent par une possibilité d'intervention des participants, selon différentes modalités (voir tableau 2).

Tableau 2. Profil délibératif des participants (réponses en \%)

\begin{tabular}{|c|c|c|c|c|c|c|c|c|}
\hline & \multicolumn{2}{|c|}{ ESPAGNE } & \multicolumn{2}{|c|}{ BRESIL } & \multicolumn{2}{|c|}{ FRANCE } & \multicolumn{2}{|c|}{ ALLEMAGNE } \\
\hline & Ferrol & Novelda & P. Alegre & Guarulhos & $\begin{array}{c}\text { Paris } \\
\left(20^{\mathrm{e}} \text { arr }\right)\end{array}$ & Grigny & Kreuzberg & Postdam \\
\hline $\begin{array}{l}\text { J'ai compris le fonction- } \\
\text { nement de la réunion }\end{array}$ & 94.1 & 95.8 & 86.7 & 84.6 & 95.2 & 96.9 & 75.0 & 92.0 \\
\hline $\begin{array}{l}\text { J'ai participé au débat } \\
\text { en demandant la parole } \\
\text { et en intervenant }\end{array}$ & 35.0 & 24.5 & 16.8 & 37.7 & 57.1 & 46.7 & 41.0 & 38.1 \\
\hline J'ai fait des propositions & 67.8 & 52.5 & 28.9 & 44.3 & 23.8 & 23.4 & 50.1 & 55.9 \\
\hline $\begin{array}{l}\text { J'ai contribué à } \\
\text { détailler ou compléter } \\
\text { une proposition faite } \\
\text { par quelqu'un d'autre }\end{array}$ & 42.9 & 46.4 & 44.3 & 51.7 & 38.1 & 39.1 & 28.6 & 26.0 \\
\hline $\begin{array}{l}\text { Jai encouragé d’autres } \\
\text { participants à faire des } \\
\text { propositions }\end{array}$ & 67.2 & 60.8 & 58.4 & 76.7 & 28.6 & 43.8 & 40.5 & 38.0 \\
\hline
\end{tabular}

$\mathrm{Ne}=918$

Source : IESA [E1106] 
Les données indiquent une intensité délibérative variable selon les cas. Un point commun se dégage toutefois : dans tous les cas, la quasi-totalité des participants affirme avoir compris le fonctionnement des assemblées. Les différences sont en revanche très fortes concernant les autres données. Afin de faciliter l'analyse et l'interprétation des données relatives à l'intensité délibérative, nous avons conçu un indice synthétique de délibération. Cet indice vise à mesurer les différentes formes de participation dans le cadre des assemblées citoyennes, en agrégeant les réponses de chaque enquêté aux cinq questions qui renvoient aux différentes modalités possibles de délibération len attribuant 0 pour une réponse négative et 1 pour une réponse positivel. L'indice varie ainsi de 0 (réponse négative à toutes les questions) à 5 (réponse positive à toutes les questions) ${ }^{3}$.

Le graphique 1 indique les moyennes obtenues grâce à notre indice synthétique pour chaque cas.

\section{Graphique 1. Moyenne de l'indice synthétique de délibération des participants dans les villes étudiées}

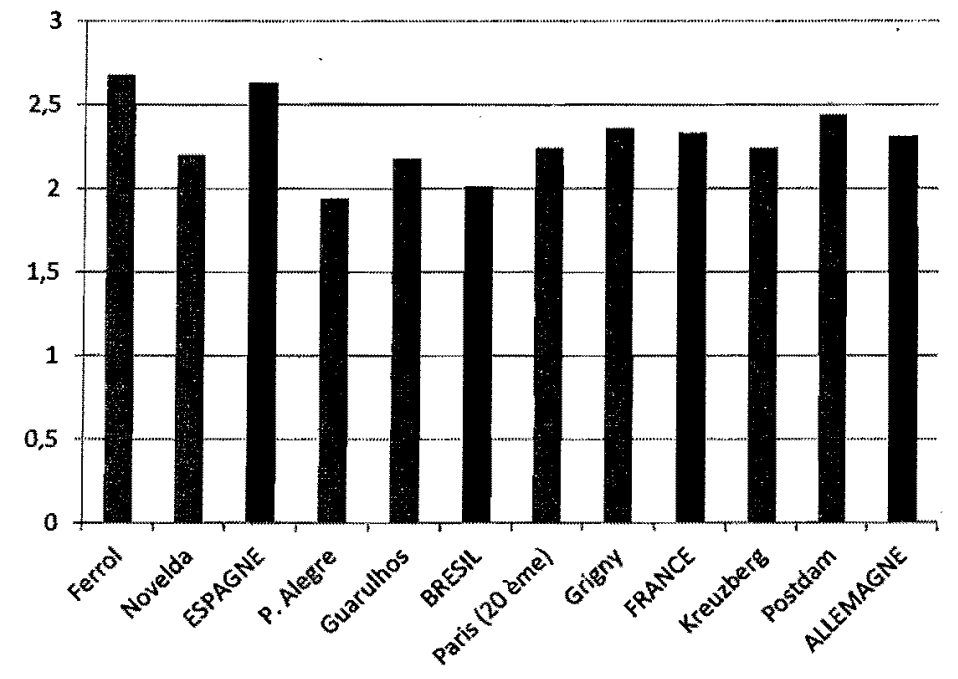

$N=918$

Source : IESA $(E \uparrow 106)$

[3] Avant d'être appliqué aux différents cas, cet indice synthétique de délibération a été testé afin de confirmer sa fiabilité et la consistance interne desitems. Les items étant des variables nominales dichotomiques (avez-vous ou non réalisé l'action en question), le critère de mesure retenu a été la méthode des deux moitiés. Pour cela, l'échelle a été décomposée en deux parties équivalentes en parallète dans le but de confirmer la consistance des items, afin d'opérer de manière analogue au mode de construction de l'intensité délibérative. Le résultat du coefficient de Spearman-Brown est de 0.552 , ce qui indique une fiabilité significative dans la pertinence de l'application de l'échelle. 
as. Un point des particidifférences de faciliter rative, nous nesurer les jyennes, en nvoient aux ne réponse onse néga$s)^{3}$.

jynthétique
Les écarts entre les cas étudiés ne sont pas particulièrement significatifs. Mais il est néanmoins intéressant de souligner que la moyenne des cas espagnols est de 2,46, celle des cas français est de 2,33 et celle des cas allemands de 2,31, alors que celle des cas brésiliens est inférieure, se situant à 2,05 . Ces différences peuvent être expliquées à partir de deux types de facteurs.

Lintensité délibérative est reliée, tout d'abord, au profil des participants. Comme nous l'avons déjà indiqué, le budget participatif conduit à des formes d'autosélection importantes liées au profil socioéconomique et sociopolitique des participants. Les personnes intéressées par la politique étant surreprésentées parmi les participants des cas allemands et français, il n'est pas étonnant que les niveaux d'intensité délibérative soient plus forts, même si les dispositifs participatifs ne sont que consultatifs.

Il est possible, ensuite, de relier l'intensité de la délibération au type de dispositif délibératif mis en place. Dans les cas espagnols, dans lesquels l'indice de délibération est le plus élevé, les participants présentent, débattent et priorisent les propositions au cours des réunions, ce qui conduit à une intensité de la délibération plus marquée. À Porto Alegre, où l'intensité de la délibération est la plus faible, les participants ne font que voter sur les propositions. Celles-ci se font en amont, et leur priorisation en aval. Dans les cas allemands et français, des débats ont également lieu dans les réunions, mais contrairement aux cas s'apparentant au modèle de la démocratie participative en Espagne et au Brésil, les décisions ne sont pas prises suite à un vote - à l'exception de Grigny.

Si on reprend les trois modèles évoqués plus haut ldémocratie participative, modernisation participative et démocratie de proximitél, leur lien avec l'indice de délibération n'est pas homogène. Au-delà des différences qui peuvent être expliquées par le profil des participants, en particulier pour les cas allemands et français, une autre explication, contre-intuitive, peut être proposée. Les données montrent qu'il n'y a pas de corrélation entre le fait qu'il s'agisse d'un dispositif délibératif dans lequel il existe, ou pas, une véritable possibilité d'influencer les décisions collectives et l'intensité de la délibération. Ce qui compte ici, c'est l'existence d'un espace destiné au débat. À partir du moment où il existe effectivement un espace pour débattre, l'intensité délibérative est similaire entre des expériences qui suivent des modèles participatifs plus restrictifs (Postdam et le $20^{\mathrm{e}}$ arrondissement de Parisl et celles qui s"inspirent de modèles plus démocratiques (Grigny, Ferrol, Guarulhos).

Il est néanmoins important de rappeler que nous analysons ici seulement la participation dans les assemblées ouvertes, où chacun est invité à participer, et pas le dispositif participatif dans son ensemble. De ce fait, nous négligeons la participation qui peut avoir lieu au sein de structures créées spécifiquement pour discuter plus en détail des différentes propositions, comme cela est le cas à Porto Alegre et dans les villes espagnoles. De ce point de vue, il paraît logique qu'au-delà des différences dans les objectifs et les procédures, les participants 
aient une intensité délibérative très semblable dans toutes les expériences, sauf dans celle de Porto Alegre où l'assemblée n'est qu'un lieu de vote et non de proposition ou d'argumentation.

Si l'intensité délibérative est semblable pour toutes les expériences qui mettent en place des lieux de débat, on peut alors se demander si les biais relatifs à l'inclusion des participants se retrouvent également dans les formes et l'intensité de la délibération dans les assemblées. Est-ce que les citoyens d'âge intermédiaire, ayant un niveau de formation plus avancé et un intérêt plus marqué pour la politique délibèrent davantage ? Cette question est centrale car, comme nous l'avons déjà mentionné, la distribution des opportunités délibératives entre les " participants est un révélateur essentiel de la qualité du processus délibératif. Le tableau 3 rassemble les données disponibles sur cette question et permet d'émettre plusieurs interprétations.

L'impact des variables sociodémographiques sur la participation, qui produisaient des formes d'auto-exclusion, ne se retrouve pas en ce qui concerne la délibération. Le critère du sexe n'est plus une variable discriminante pour la délibération dans la plupart des cas, même s'il a davantage d'influence dans les cas brésiliens et espagnols. Les critères de l'âge et du niveau de formation cessent de produire des effets. L'impact de ces facteurs sur la délibération n'est pas significatif statistiquement. Même si les participants les plus jeunes ou avec un niveau de formation plus faible sont les moins nombreux, on n'observe pas de différence d'intensité délibérative entre les participants une fois le débat commencé.

En ce qui concerne l'impact des variables sociopolitiques, les résultats confirment en revanche l'asymétrie observée pour la participation, même si des différences importantes existent entre les pays. Lintérêt pour la politique et la participation à des associations renforcent cette asymétrie dans les cas espagnols et brésiliens, où l'engagement associatif par exemple influence positivement l'intensité délibérative dans presque tous les cas. Cela semble indiquer qu'en Espagne et au Brésil les associations jouent en général un rôle plus important dans les processus participatifs. En revanche, les cas allemands et français présentent une forte hétérogénéité des facteurs significatifs - les variables de l'intérêt pour la politique et de l'expérience antérieure dans les processus participatifs étant celles qui influencent le plus l'intensité délibérative des participants. Ces différences peuvent conduire à penser qu'il existe deux groupes aux comportements distincts, d'une part les cas brésiliens et espagnols, et, de l'autre, les cas français et allemands. Le tableau 4 présente l'influence de différentes variables, en regroupant les cas suivant ces deux groupes. 
Tableau 3. Influence des variables sociodémographiques et sociopolitiques dans la délibération au sein du budget participatif. Différences dans les moyennes de l'indice synthétique de délibération selon les variables. Répartition par villes

\begin{tabular}{|l|c|c|c|c|c|c|c|c|}
\hline & \multicolumn{2}{|c|}{ ESPAGNE } & \multicolumn{2}{c|}{ BRÉSIL } & \multicolumn{2}{c|}{ FRANCE } & \multicolumn{2}{c|}{ ALLEMAGNE } \\
\hline & Ferrol & Novelda & P. Alegre & Guarulhos & Paris (20 arr. & Grigny & Kreuzberg & Postdam \\
\hline SEXE & & & & & & & & \\
\hline Homme & 2,92 & 2,28 & 1,86 & 2,48 & 2,23 & 2,79 & 2,06 & 2,53 \\
\hline Femme & 2,49 & 2,24 & 1,98 & 1,91 & 2,25 & 1,70 & 2,37 & 2,21 \\
\hline F de ANOVA & 4,863 & 0,037 & 0,236 & 4,871 & 0,001 & 8,631 & 0,898 & 0,571 \\
\hline ÂGE & & & & & & & & \\
\hline Jusqu'à 24 & 3,44 & 2,05 & 1,77 & 1,88 & & 2,50 & 2,00 & 2,00 \\
\hline $25-44$ & 2,56 & 2,52 & 2,04 & 2,22 & 2,67 & 2,25 & 2,24 & 2,59 \\
\hline 45-65 & 2,67 & 2,18 & 1,83 & 2,25 & 2,27 & 2,78 & 2,23 & 2,21 \\
\hline Plus de 65 & 2,71 & 1,73 & 2,3 & 2,25 & 1,60 & 1,71 & 2,40 & 2,55 \\
\hline F de ANOVA & 1,119 & 1,632 & 0,747 & 0,118 & 0,989 & 1,593 & 0,081 & 0,305 \\
\hline NIVEAU DEFORMATION & & & & & & & & \\
\hline Primaire non achevée & 2,22 & 1,93 & 1,77 & 2,5 & & 1,00 & 2,00 & 1,50 \\
\hline Primaire & 2,4 & 2,15 & 2,6 & 2,08 & & 3,10 & 2,00 & 2,60 \\
\hline Secondaire & 3,01 & 2,21 & 1,93 & 1,86 & 2,17 & 2,39 & 2,28 & 2,17 \\
\hline Supérieure & 2,58 & 2,67 & 1,91 & 2,63 & 2,36 & 2,14 & & 2,50 \\
\hline Fde ANOVA & 2,526 & 1,935 & 0,137 & 1,955 & 0,528 & 1,105 & 0,326 & 0,485 \\
\hline $\begin{array}{l}\text { AUTOPOSITIONNEMENT } \\
\text { IDÉOLOGIQUE }\end{array}$ & & & & & & & & \\
\hline Gauche & 2,73 & 2,48 & 2,12 & 2,67 & 2,00 & 2,62 & 2,23 & 2,36 \\
\hline Centre & 2,7 & 1,94 & 1,86 & 2,1 & 2,40 & 2,33 & 1,89 & 2,40 \\
\hline Droite & 2,48 & 2,15 & 2,02 & 2,41 & 2,75 & 1,63 & 1,00 & 2,00 \\
\hline F de ANOVA & 0,319 & 2,543 & 0,493 & 1,369 & 0,511 & 1,348 & 0,615 & 0,154 \\
\hline
\end{tabular}




\begin{tabular}{|l|c|c|c|c|c|c|c|c|}
\hline $\begin{array}{l}\text { INTERETT POUR } \\
\text { LAPOLITIQUELOCALE }\end{array}$ & & & & & & & & \\
\hline Beaucoup/Assez & 2,84 & 2,51 & 1,48 & 2,28 & 2,30 & 2,46 & 2,41 & 2,42 \\
\hline Peu/Aucun & 2,39 & 1,94 & 2,08 & 1,65 & 1,00 & 1,71 & 1,60 & 3,00 \\
\hline F de ANOVA & 3,694 & 6,359 & 6,006 & 2,491 & 1,013 & 1,435 & 4,343 & 0,375 \\
\hline $\begin{array}{l}\text { INTÉRÉT POUR } \\
\text { LA POLITIQUE NATIONALE }\end{array}$ & & & & & & & & \\
\hline Beaucoup/ASSEz & 2,83 & 2,5 & 1,56 & 2,38 & 2,41 & 2,59 & 2,24 & 2,43 \\
\hline Peu/Aurcun & 2,49 & 2,02 & 2,14 & 1,56 & 1,50 & 1,50 & 2,25 & 2,50 \\
\hline Fde ANOVA & 1,950 & 4,133 & 6,685 & 3,899 & 0,884 & 4,238 & 0,001 & 0,014 \\
\hline $\begin{array}{l}\text { EXPÉRIENCE } \\
\text { DANSLEPROCESSUS }\end{array}$ & & & & & & & & \\
\hline Non & 2,5 & 2,14 & 1,47 & 1,6 & 2,10 & 1,36 & 1,98 & 1,79 \\
\hline Oui & 3,05 & 2,74 & 2,26 & 2,8 & 2,50 & 2,57 & 2,77 & 2,84 \\
\hline Fde ANOVA & & 6,747 & 14,234 & 24,453 & 0,490 & 6,023 & 6,587 & 8,724 \\
\hline $\begin{array}{l}\text { PARTICIPATION } \\
\text { A DES ASSOCIATIONS }\end{array}$ & 2,23 & 1,86 & 1,32 & 1,46 & 1,00 & 2,38 & 1,93 & 2,27 \\
\hline Non & 2,79 & 2,26 & 2,1 & 2,67 & 2,37 & 2,36 & 2,35 & 2,49 \\
\hline Oui & 5,657 & 2,821 & 8,873 & 24,622 & 2,265 & 0,001 & 1,395 & 0,226 \\
\hline Fde ANOVA & & & & & & & \\
\hline
\end{tabular}

En gras : différences significatives pour $p<0,05$

$N=918$

Source: IESA (E1106) 
Tableau 4. Influence des variables sociodémographiques et sociopolitiques dans la délibération. Différences des moyennes de l'indice synthétique de délibération selon différentes variables.

Distribution par mödèles

\begin{tabular}{|c|c|c|}
\hline & ESPAGNE/BRÉSIL & FRANCE/ALLEMAGNE \\
\hline SEXE & & \\
\hline Homme & 2,43 & 2.46 \\
\hline Femme & 2,16 & 2,17 \\
\hline F de ANOVA & 4,635 & 2.266 \\
\hline$\hat{A} G E$ & & \\
\hline Jusqu'à 24 & 2,34 & 2.11 \\
\hline $25-44$ & 2,41 & 2,34 \\
\hline $45-65$ & 2,41 & 2.41 \\
\hline Plus de 65 & 2,47 & 2,06 \\
\hline F de ANOVA & 0.060 & 0,615 \\
\hline NIVEAU DE FORMATION & & \\
\hline Sans formation & 2.00 & 2,00 \\
\hline Primaire non terminée & 2,08 & 1.80 \\
\hline Primaire & 2.25 & 2,63 \\
\hline Secondaire & 2,32 & 2,30 \\
\hline Supérieure & 2,40 & 2,38 \\
\hline FdeANOVA & 1,015 & 0.582 \\
\hline POSITIONNEMENTIDÉOLOGIQUE & & \\
\hline Gauche & 2,54 & 2,34 \\
\hline Centre & 2,10 & 2,25 \\
\hline Droite & 2,22 & 1.94 \\
\hline F de ANOVA & 6,010 & 0.639 \\
\hline INTÉRÊT POUR LA POLITIQUE LOCALE & & \\
\hline Beaucoup/Assez & 2,44 & 2,41 \\
\hline Peu/Aucun & 2,07 & 1,72 \\
\hline$F$ de ANOVA & 9,831 & 5.528 \\
\hline INTÉRÊT POUR LA POLITIQUE NATIONALE & & \\
\hline Beaucoup/Assez & 2,44 & 2,39 \\
\hline Peu/Aucun & 2,12 & 1.96 \\
\hline Fde ANOVA & 6,791 & 2.149 \\
\hline EXPÉRIENCE DANS LE PROCESSUS & & \\
\hline Non & 2.02 & 1,89 \\
\hline Oui & 2,64 & 2,68 \\
\hline Fde ANOVA & 32,068 & 18.921 \\
\hline PARTICIPATION ASSOCIATIVE & & \\
\hline Non & 1.71 & 2.07 \\
\hline Ouĩ & 2,48 & 2,39 \\
\hline F de ANOVA & 41,407 & 1.873 \\
\hline
\end{tabular}

En gras : différences significatives pour $p<0,05$

$N=918$

Source : IESA [E1106 
Dans les deux groupes, les variables sociodémographiques n'influencent que marginalement la délibération au sein des assemblées citoyennes. Seul le facteur du sexe a dans le groupe Brésil/Espagne une influence sensible sur l'intensité délibérative, les hommes étant plus actifs dans la délibération. Les variables sociopolitiques ont en revanche un impact significatif sur les participants dans ces deux pays, alors que cela n'est pas le cas en France et en Allemagne. On peut ainsi estimer que plus on se dirige vers le sud, plus le participant type est un homme, de gauche, ayant un intérêt marqué pour la politique. intégré dans des activités associatives et ayant une expérience antérieure dans le budget participatif. Dans les cas français et allemands, l'intensité délibérative est principalement influencée par l'existence d'expériences antérieures de participation et l'intérêt pour la politique, mais de manière générale il semble s'agir de processus moins politisés et davantage ouverts à des citoyens non organisés.

\section{Conclusion}

Les résultats présentés dans cet article suggèrent une différence entre les deux groupes de pays. Les expériences françaises et allemandes sont plus restrictives en matière d'inclusion démocratique. Dans les cas de Postdam ou de Paris, par exemple, les biais sociodémographique et sociopolitique sont très forts en termes de participation. Les participants étant majoritairement issus des fractions supérieures des classes moyennes politisées, le biais délibératif s'en trouve diminué en nivelant les possibilités de participation. Ce biais pourrait expliquer que ces expériences sont symétriques en termes de distribution des opportunités de délibération, même s'il s'agit de modèles participatifs plus restrictifs. La majorité des participants au sein de ces expériences étant davantage socialisée à la participation, il est logique d'observer des disparités moindres en termes de délibération. Une autre explication de la moindre intensité du biais délibératif dans ces cas est l'absence de prise de décision confiée aux participants. J. Habermas signale en effet que le dialogue est dautant plus ouvert qu'il a lieu en marge de la prise de décision : une interprétation qui nous semble pertinente pour les modèles participatifs consultatifs.

Les cas espagnol et brésilien s'avèrent au contraire comparativement plus ouverts en termes de participation, mais beaucoup plus restrictifs pour ce qui est de la délibération. Cela n'est pas étranger au fait que, dans ces deux pays, les citoyens décident de l'allocation réelle de ressources publiques, ce qui renforce la place dans l'espace délibératif des participants qui ont déjà l'expérience du débat public, face à ceux qui en ont moins l"habitude. Le poids des militants associatifs s*avère ici déterminant. Ce sont eux qui au Brésil et en Espagne ont traditionnellement l'habitude de négocier les petits investissements urbains avec les représentants élus, et qui transfèrent cette expérience à un espace désormais plus formalisé. 
s n'influencent que citoyennes. Seul le vence sensible sur la délibération. Les icatif sur les partias en France et en e sud. plus le partijé pour la polítique, ice antérieure dans 'intensité délibéraices antérieures de générale il semble à des citoyens non

ifférence entre les ndes sont plus resde Postdam ou de politique sont très joritairement issus le biais délibératif n. Ce biais pourrait de distribution des rticipatifs plus reses étant davantage isparités moindres e intensité du biais confiée aux partiautant plus ouvert on qui nous semble

parativement plus trictifs pour ce qui ans ces deux pays, liques, ce qui renIt déjà l'expérience zoids des militants et en Espagne ont ssements urbains ience à un espace
Ces résultats sont ambivalents concernant la question de l'impact et de la portée délibérative des budgets participatifs : ils montrent un processus participatif effectivement orienté par la délibération, où le débat public se déroule dans un espace formel, à l'inverse du modèle communicationnel habermassien.

Le contenu délibératif du budget participatif est néanmoins conditionné ici par le profil des participants et contraint par les asymétries participatives qui lui préexistent dans la société. Le budget participatif fait en réalité participer des personnes dont le profil est majoritairement celui de citoyens déjà actifs, motivés et qui ont une expérience de la délibération avec des représentants élus dans d'autres arènes. Le budget participatif incarne un canal alternatif de participation, mais celui-ci est en réalité nourri essentiellement par des citoyens qui sont déjà actifs en dehors de lui, et qui s insèrent dans ce nouvel espace pour chercher à influencer les décisions des assemblées sur les décisions publiques. Même si dans ce nouveau modèle, les associations renoncent à leur rôle de médiation, elles occupent de fait une position privilégiée dans le budget participatif du fait du capital social de leurs membres. Malgré cela, le budget participatif parvient à agir sur, ou du moins à estomper, les asymétries d'intensité délibérative liées à l'âge ou au niveau de formation auxquelles on pourrait s'attendre, et qui ont déjà été soulignées dans les travaux académiques sur la participation. Les expériences étudiées ici montrent en effet qu une fois les participants présents dans les dispositifs, les différences relatives à ces variables n'ont quasiment plus d'effet sur leur intensité délibérative.

Nous pouvons également observer des différences dans l'intensité délibérative selon les pays. En règle générale, le fait qu'une personne ait participé par le passé à un dispositif participatif explique en grande partie quelle intervienne dans le débat de manière plus importante que les autres. On peut néanmoins penser que la procédure concrète du dispositif participatif importe moins que le modèle structurel dans lequel s’insère la délibération. Lintensité délibérative est très similaire dans toutes les expériences étudiées, indépendamment de leur caractère consultatif ou contraignant vis-à-vis des élus. L'expérience de Porto Alegre contraste fortement avec celle de Postdam. Alors que la première constitue la référence participative dans l'imaginaire des spécialistes de la question, à Postdam, bien que le dispositif ne soit pas relié directement à la décision, les participants délibèrent beaucoup plus. Labsence d'assemblée délibérative dans le cas de Porto Alegre apparaît comme l'élément explicatif central, ce qui n'empêche pas de questionner également les points communs entre les dynamiques nationales.

Loriginalité de l"invention brésilienne a dépassé toutes les attentes. L'histoire de sa diffusion montre qu'il s'agit d'un instrument qui a été mis en ceuvre dans des contextes et des cultures très différents (Sintomer et al., 2010). Les résultats indiquent des ressemblances entre les deux groupes de pays, dont la configuration diffère de ce à quoi on pouvait s'attendre. Il n'y a pas en effet de spécificité des cas brésiliens ou européens, car les expériences au Brésil et en Espagne partagent 
plus de points communs entre elles quavec les cas français ou allemands. Les spécificités institutionnelles ou économiques de chaque pays ne seraient ainsi pas un obstacle au développement du budget participatif, alors que les caractéristiques sociales ou culturelles auraient une incidence plus importante. L'Espagne et le Brésil partagent le fait d'avoir connu des régimes dictatoriaux dont l'effondrement a donné lieu à des régimes démocratiques nouveaux, respectivement en 1975 et 1985. Dans ces deux cas, les mouvements sociaux urbains ont fait pression de manière significative pour obtenir une démocratisation des pouvoirs locaux, et leur présence dans les villes aujourd'hui continue à être une caractéristique importante de la gouvernance urbaine. Contrairement à ce qui s'est passé en France et en Allemagne, la place des mouvements sociaux urbains dans la démocratisation des espaces politiques municipaux a été permanente dans les trente dernières années. La mise en œuvre des budgets participatifs ne peut se comprendre dans ces cas sans leur présence, quand bien même ces dispositifs ont été décidés par les pouvoirs politiques locaux, et souvent contre les mouvements sociaux comme à Porto Alegre (Baiocchi, 2005) ou à Cordoue en Espagne [Ganuza, Francés, 2012]. D'une certaine manière, et en étant attentifs à ne pas généraliser de façon trop hâtive, nous pourrions penser que le contexte participatif urbain influence fortement ces dynamiques. Au Brésil comme en Espagne, les processus participatifs sont d'autant plus inclusifs qu'il existe un mouvement urbain dynamique. Au vu de l'importance qu'acquiert dans la participation le fait d'être membre d'une association locale, it est certain qu'une plus forte densité associative peut aider. Néanmoins, les dynamiques internes de ce modèle sont plus élitistes, ce qui reflète l'histoire des mouvements sociaux urbains, marqués par une structuration hiérarchisée, un faible renouvellement générationnel, et dont les représentants dominent la plupart des instances participatives municipales dans les pays en question. Au contraire, les processus français et allemands sont moins inclusifs, mais moins élitistes dans leur dynamique interne, reflétant ainsi une culture politique différente.

Toutefois, dans tous les cas, la structuration du débat public est confrontée à des défis considérables liés au recrutement des participants. Linfluence du biais socioéconomique et socioéducatif est particulièrement forte et a toujours été soulignée dans la littérature sur la participation. Le tournant délibératif a raison de souligner que ce biais peut être corrigé par une interaction communicationnelle. Nos données confirment cette hypothèse, car ni la formation ni l'âge ne sont des facteurs qui limitent le fait d'intervenir et de parler dans les assemblées. Ce qui importe, c'est bien l'existence d'un espace délibératif, et les participants font ensuite le reste. Notre étude ne permet pas d'analyser la qualité détibérative en termes substantifs, comme cela a été fait dans d'autres travaux à caractère plus ethnographique sur la délibération et les dynamiques des budgets participatifs. Julien Talpin (2011) a ainsi analysé la délibération en suivant une telle perspective, par la comparaison de trois cas de budgets participatifs. Selon ses travaux, la qualité délibérative était faible, mais elle augmentait lorsque les procédures el les thématiques de discussion permettaient l'émergence d'une forme de conflictualité. Une enquête par questionnaire ne permet 
nands. Les ntainsipas :aractéris. L'Espagne unt l'effonzctivement ns ont fait s pouvoirs ine carace qui s'est $1 \times$ urbains armanente articipatifs même ces ent contre ordoue en attentifs à a contexte :omme en existe un ns la parjin quiune $s$ internes ts sociaux vellement inces parrocessus eur dyna-

Ifrontée à uence du i toujours ibératif a commumation ni dans les jératif, et ralyser la ; d'autres ramiques ration en $s$ particigmentait t l'émere permet pas de connaître la qualité et le poids relatif des arguments, mais seulement de savoir si les personnes présentes ont participé au débat, si elles ont présenté une proposition, si elles ont voté. C'est pour cela que nous parlons d'intensité délibérative, et à partir de l'analyse de nos données, nous pouvons en déduire que l'espace délibératif est en réalité plus ouvert que ce que signale habituellement la littérature académique sur la participation et la délibération. Nous pouvons penser qu'un espace plus ouvert en termes de participation permet une extension des thématiques discutées, ce qui peut conduire par conséquent à une plus grande conflictualité de la discussion, due à la présence de points de vue plus diversifiés dans le débat. Notre étude donne ainsi à voir un espace dans lequel la distribution des opportunités délibératives est moins inégalitaire qu'on pourrait le penser a priori.

La participation est confrontée à des défis relatifs à l'inclusion démocratique, qui ne sont pas facilement solubles. La littérature académique a beaucoup insisté sur le comportement politique des personnes qui participent, en en déduisant que ces dispositifs constituent un cadre politique d'apprentissage pour les personnes qui ne participent pas habituellement. Nous ne sommes pas certains que cette stratégie puisse réduire le problème du recrutement : dans le processus délibératif, la réduction des asymétries montre que ces dispositif́s génèrent des éléments positifs pour les citoyens, dans lesquels les différences substantielles entre eux ne sont plus définitives. Partant de là, de nouveaux questionnements sur cette manière de structurer la politique apparaissent.

Les gouvernements locaux ont une forte capacité à instrumentaliser les espaces participatifs quand le recrutement des participants dépend d'eux. Cela ne veut pas dire que ce contrôle soit systématique, d'autant que l'on retrouve très fréquemment les biais habituels de la participation politique. Même si l'on prend en compte qu'il s'agit d'espaces au sein desquels des décisions sont prises sur l'allocation des biens publics - ou du moins des consultations sont réalisées -. l'ouverture effective des espaces participatifs demeure un objectif essentiel. Cette ouverture, pensée de telle façon à ce quiun maximum de personnes se sentent invitées à participer [Verba et al., 1995], ne dépend pas seulement du profil individuel des participants, car l'effet éventuel de ces différences s'estompe au moment de la délibération. Mais il est nécessaire de créer un cercle vertueux reliant la participation et la délibération à leurs effets politiques (Avritzer, 2006 ; Ganuza, Francés, 2012]. Le cas portugais est ici très révélateur, car dans les dix dernières années les seules expériences qui ont survécu sont précisément celles ayant eu des effets directs sur les décisions publiques (Lopes Alves. Allegretti, 20121. Cela semble constituer un antidote à l'instrumentalisation de ces dispositifs. Les citoyens cessent de participer sils comprennent que le processus camoufle une absence de projet politique, et au contraire ils appuient les budgets participatifs s'ils voient concrètement les effets de leur participation au-delà des réunions auxquelles ils ont assisté. 


\section{Bibliographie}

Avritzer L., 2006. « New Public Spheres in Brazil: Local Democracy and Deliberative Democracy », International Journal of Urban and Regional Research, 30 [3], p. 623-639.

Baiocchi G., 2005, Militants and Citizens, Standford, Standford University Press.

Baiocchi G., Ganuza E., 2014. « Participatory Budgeting: as if Emancipation Mattered ", Politics \& Society, 42 (1), p. 19-50.

Barabas J., 2004, « How Deliberation affects policy Opinions », American Political Science Review, 98 [4], p. 687-701.

Bryan F., 2004, Real Democracy: the New England Town Meeting and How it Works, Chicago, Chicago University Press.

Eliasoph N., 1998, Avoiding Politics: How Americans Produce Apathy in Everyday Life, Cambridge/New York, Cambridge University Press.

Fiorina M. P., 1999, « Extreme Voices: a Dark Side of Civil Engagement », in T. Skocpol, M. P. Fiorina Idir.J, Civic Engagement in America Democracy, Washington DC, Brookings Institutions Press, p. 395-426.

Ganuza E., Frances F., 2012, El círculo virtuoso: los presupuestos participativos a debate, Madrid, CIS.

Goldfrank B., 2012, «The World Bank and the Globalization of Participatory Budgeting ", Journal of Public Deliberation, 8 (2), http:/www.publicdeliberation.net/jpd/vol8/iss2/art7.

Habermas J., 1996. Between Facts and Norms: Contributions to a Discourse Theory of Law and Democracy, Cambridge, MIT Press.

Herzberg C., 2011, « Democratic Innovation or Symbolic Participation? Participatory Budgeting in Germany », Paper presented for 6th ECPR General Conference Panel 25 - Democratic Innovations in Europe - A comparative perspective 25th - 27th Aug. 2011, Reykjavik, Iceland.

Hibbing J. R., Theiss-Morse E., 2002, Stealth Democracy: Americans' Beliefs About How Government Should Work, Cambridge, Cambridge University Press.

Lopes Alves M., Allegretti G., 2012, « (In)stability, a Key Element to Understand Participatory Budgeting : Discussing Portuguese Cases », Journal of Public Deliberation, 8 (2), http://www.publicdeliberation.net/jpd/vol8/iss2/art3.

Mutz D., 2006, Hearing the Other Side, Cambridge/New York, Cambridge University Press.

Nez H., 2010, Les savoirs citoyens dans l'urbanisme participatif : regards croisés sur les expériences de Paris et Cordoue, Thèse de doctorat en sociologie, Université Paris 8. Universidad Autónoma de Barcelona.

Sanders L., 1997, « Against Deliberation », Political Theory, 25, p. 347-376.

Sintomer Y., Herzberg C., Röcke A., 2008, Les budgets participatifs en Europe, Paris, La Découverte.

Sintomer Y., Herzberg C., Allegretti G., 2010, Learning from the South: Participatory Budgeting Worldwide, Bonn, InWEnt gGmbH. 
ocracy and 7d Regional

ity Press.

nancipation

can Political

ow it Works,

in Everyday

iment $»$, in Democracy,

ticipativos a

Irticipatory icdelibera-

Irse Theory

ticipation? ¿R General mparative

:liefs About ress.

inderstand al of Public lart3.

University

croisés sur Université

176.

?n Europe, rticipatory
Swyngedouw E., 2005, « Governance Innovation and the Citizen: the Janus Face of Governance-Beyond-the-State $»$, Urban Studies, 42 [11], p. 1991-2006.

Talpin J., 2011, Schools of Democracy: How Ordinary Citizens (Sometimes) Become Competent in Participatory Budgeting Institutions, Colchester, ECPR Press.

Verba S., Schlozman K. L, Brady H. E., 1995, Voice and Equality. Civic Voluntarism in American Politics, Cambridge, Cambridge University Press.

Wampler B., 2007, « Can Participatory Institutions Promote Pluralism? Mobilizing Low-Income Citizens in Brazil ». Studies in Comparative International Development, 41 (4), p. 57-79.

\section{Remerciements}

Les auteurs remercient les commentaires des évaluateurs de la revue, ainsi que ceux des éditeurs. Cet article a été réalisé dans le cadre diun projet financé par le Ministère espagnol de ['Économie et de la Compétitivité [CS02012-38942]

\section{Abstract - The Participatory Challenge: Deliberation and Democratic Inclusion in Participatory Budgeting}

Participatory theory has undergone a communicative shift which has, during recent years, favoured the organisation of new participatory experiments based on classic principles of deliberative theory, such as the principle of inclusion and the equality of individuals with regard to the act of deliberating. The development of participatory budgets over the last decade provides an excellent analytical framework for finding out how the experiments implement the principle of inclusion and to what extent they allow participants to effectively take advantage of the opportunities for deliberation. This paper attempts to contextualize this process, what it has meant and how t has taken place, making use of a comparative research between the experiences of Brazil, Spain, France and Germany. The question tries to frame an emergent discussion about the conflict or existing challenge between the deliberative turn of the political theory and participation is is understood that the deliberation demands spaces of reflection that participatory p rocesses does not contemplate. Participatory budgeting, however, aims to merge both dimensions our purpose is to understand how and what are the differences that emerge in different political contexts. The results allow to better understand how to work the various procedures mplemented and what is the relationship between deliberation and participation.

Keywords participator y budgeting, participative democracy participatory modernisation, local democracy, inclusion, deliberation 
Ernesto Ganuza est chercheur en sociologie au sein du Centre de la Recherche Scientifique Espagnol (IESA/CSIC). Il a fait ses études à Madrid, avant d'être chercheur invité à Berlin [Centre Marc Bloch], à Paris [CNRS] et Providence [Université de Brown]. II travaille sur la démocratie, les budgets participatifs et la société civile. Il s'intéresse notamment aux espaces publics de participation et aux défis de démocratisation qui découlent des rapports de pouvoir en leur sein. Il a publié différents articles et ouvrages sur le sujet. eganuzalaiesa.csic.es

Prix spécial de thèse en sociologie de l'Université d'Alicante, Francisco Francés est aujourd'hui maître de conférences au sein du département de sociologie et chercheur à Institut Interuniversitaire pour le Développement Social et la Paix. Ses travaux portent sur la participation, la sociologie politique, l'immigration et la jeunesse. En tant que chercheur, il a collaboré avec différentes institutions nationales et internationales, et participé à plus vingt projets publics. Il est l'auteur de plusieurs livres et articles publiés dans des revues internationales sur la question de la participation et du budget participatif. Il a également animé divers processus de participation au niveau local. francisco.francesđuua.es

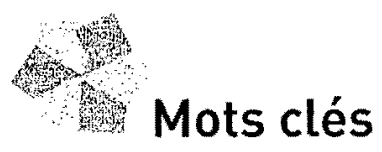

budget participatif, démocratie participative, modernisation participative, démocratie locale, inclusion, délibération 